\title{
BMJ Open Quality Improving lung function in adolescents with CF by tracking annual rate of lung function decline
}

\author{
Sydney Schiff, ${ }^{1,2}$ Miah Starks, ${ }^{1}$ Rachel W Linnemann ${ }^{1,3}$
}

To cite: Schiff S, Starks M, Linnemann RW. Improving lung function in adolescents with CF by tracking annual rate of lung function decline. BMJ Open Quality 2021;10:e001199. doi:10.1136/ bmjoq-2020-001199

- Additional supplemental material is published online only. To view, please visit the journal online (http://dx.doi.org/10. 1136/bmjoq-2020-001199).

This work was presented as a poster presentation at the North American Cystic Fibrosis Conference, Denver, Colorado, USA, in October 2018.

Received 8 September 2020 Accepted 25 July 2021

\section{ABSTRACT}

Background For patients with cystic fibrosis (CF), sustaining lung function through the adolescent years is crucial to slow the progressive decline that leads to significant morbidity and early mortality. This holds true for patients with high per cent predicted forced expiratory volume in $1 \mathrm{~s}\left(\mathrm{ppFEV}_{1}\right)$, as they may receive less vigilant monitoring and treatment. Early identification of lung function decline followed by aggressive treatment can lead to preservation of lung function.

Intervention The Emory+Children's Pediatric Cystic Fibrosis Program implemented multiple quality improvement (QI) initiatives to identify and aggressively treat adolescent patients with a rapid decline in lung function. These initiatives included (1) lung zones to categorise and highlight lung function decline, (2) individual lung decline tables for quick reference, (3) a lung health algorithm to encourage uniformity, (4) a rapid decliner checklist to identify potential reasons for individual decline and (5) an automated individual patientlevel data report and centre scorecard. We tested these interventions with plan-do-study-act cycles and refined as needed.

Results Implementation of these QI initiatives resulted in overall improvement in lung function and slowing of lung function decline among adolescents with $\mathrm{CF}$. This improvement could be attributed to the more standardised and proactive approach to decreases in lung function and the increased clinician attention to patients with rapid decline, especially for patients with high baseline $\mathrm{ppFEV}_{1}$.

\section{INTRODUCTION}

Cystic fibrosis (CF) is a chronic, life-limiting genetic condition that leads to progressive lung disease over time. Mutations in the cystic fibrosis transmembrane conductance regulator (CFTR) gene cause mucus in the airways to be dehydrated, leading to various complications including infections, inflammation, lung injury and respiratory failure. ${ }^{1}$ The clinical course is also characterised by acute pulmonary exacerbations, or intermittent worsening of signs and symptoms that are often accompanied by a decline in lung function. ${ }^{2}$ It is vital to identify and treat these exacerbations as early as possible to slow the progression of lung disease, as they have a significant impact on mortality, ${ }^{3}$ quality of life ${ }^{4}$ and long-term lung function. ${ }^{5}$ Consistent and aggressive treatment of pulmonary exacerbation is associated with improved outcomes and preserving lung function. ${ }^{6} 7$ Additionally, progressive CF pulmonary disease may lead to steady decline in lung function over time. This decline may be more clinically challenging to identify but also results in advancing lung disease.

Although life expectancy is increasing for patients with $\mathrm{CF}$, there remains a mortality peak in early adulthood due to progressive pulmonary disease with respiratory failure. ${ }^{8}$ This pulmonary decline may be slowed with aggressive monitoring of per cent predicted forced expiratory volume in $1 \mathrm{~s}\left(\mathrm{ppFEV}_{1}\right)$ and intervention during the paediatric and adolescent years. Patients with a high baseline $\mathrm{ppFEV}_{1}$ may receive less vigilant monitoring despite having rapid rates of lung function decline. ${ }^{910}$

Data from the Emory+Children's Pediatric Cystic Fibrosis Program showed belowaverage lung function in 2014 and 2015 in our adolescent population. We undertook a quality improvement (QI) initiative to track lung function decline and implement interventions based on QI principles. Our global aim was to reduce the annual rate of decline in $\mathrm{ppFEV}_{1}$ and improve median lung function among our adolescents with CF. Our SMART aim was to improve $\operatorname{ppFEV}_{1}$ among adolescents 13-17years of age from a baseline of $86 \%$ in 2016 to $90 \%$ (above the national average of $88 \%$ ) in 3 years.

\section{METHODS}

\section{Context}

The Children's Healthcare of Atlanta and Emory University Cystic Fibrosis Care Center, located in Atlanta, Georgia, provides care for almost 700 patients with $\mathrm{CF}$ and is one of the largest specialised care centres for this disease in the USA. The centre is accredited by the Cystic Fibrosis Foundation and one of only two CF care centres in Georgia. At the time 
this QI project was initiated in 2016, the Emory+Children's Pediatric Program provided clinical care for nearly 200 children and adolescents with CF. Of these, about $47 \%$ were male and $88 \%$ were white. Of note, the percentage of black patients followed up at our programme is approximately twice the national average for paediatric programmes. The most common mutation among our patients was F508del, with nearly $90 \%$ of patients having at least one copy. F508del is the most frequently identified $\mathrm{CF}$ mutation and is a protein processing mutation that results in little to no functional CFTR channel at the cell surface and classic CF disease. ${ }^{1}$ In 2016, $24 \%$ of patients at our programme $<18$ years of age were infected with Pseudomonas aeruginosa; $21 \%$ were infected with methicillinresistant Staphylococcus aureus; and 35\% had one or more pulmonary exacerbations per year. These characteristics were fairly typical of the national CF population except for our exacerbation frequency, which was $10 \%$ higher than the national average of 25\%. Among adolescents aged 10-17years, approximately $15 \%$ had a diagnosis of CF-related diabetes (national average 13\%). CF-related diabetes is a complication associated with a more rapid rate of lung function decline and increased mortality. ${ }^{11}$ Interdisciplinary $\mathrm{CF}$ care at our clinic is provided by a team that consists of physicians, nurse practitioners, nurses, respiratory therapists (RTs), dietitians and social workers. Our programme has a strong focus on QI with biweekly full team QI meetings.

\section{Patient and public involvement}

Patients and the public were not involved in the design of these QI interventions. As part of this project, patients and families were educated about their individual baseline lung function and rate of decline. The project design and results were presented to patients and families at our annual CF education day in 2018.

\section{Interventions}

\section{Study of the interventions}

We followed the model for improvement ${ }^{12}$ methodology by using a key driver diagram (figure 1) and plan-dostudy-act (PDSA) cycles to implement and refine the following interventions to reduce the annual rate of decline in forced expiratory volume in $1 \mathrm{~s}\left(\mathrm{FEV}_{1}\right)$ and improve $\mathrm{ppFEV}_{1}$ for our adolescents with $\mathrm{CF}$.

\section{PDSA cycle 1 (January 2017)}

We created and assigned lung zones to individual adolescent patients with CF based on their rate of lung function decline over time. An individual's baseline $\mathrm{ppFEV}_{1}$ was calculated for each year as the average of the best value of each quarter, according to the method used for the CF Foundation's annual programme reports. We selected this definition to allow us to benchmark to the national averages calculated using this method. Additionally, our tracked data would match and predict the final data we received in our CF Foundation benchmarking report annually. Using each individual's yearly baseline $\mathrm{ppFEV}_{1}$, we calculated the annual rate of decline (\%) from 2013 to 2016. Patients with a history of lung transplant were excluded. A total of 69 eligible adolescents had data for the time period of 2013-2016 for whom we could calculate a 3-year rate of decline. Quarterly best $\mathrm{ppFEV}_{1}$ values were manually extracted from our pulmonary function test (PFT) software by our CF RTs and entered into a spreadsheet that calculated baseline lung function and rate of decline. Mean annual rate of decline was $-2.1 \%$ / year (SD 3.3), similar to published reports. ${ }^{13}$ However, significant variability in rates of decline was noted: $25 \%$ $(\mathrm{n}=17)$ had no decline in $\mathrm{ppFEV}_{1}$, while the bottom quartile had rates of decline of $-3.7 \%$ to $-13.3 \%$ per year. A review of risk factors for rapid decline in the initial 69 adolescents with $\mathrm{CF}$ revealed that patients with CF-related diabetes diagnosed by 2013 had significantly faster

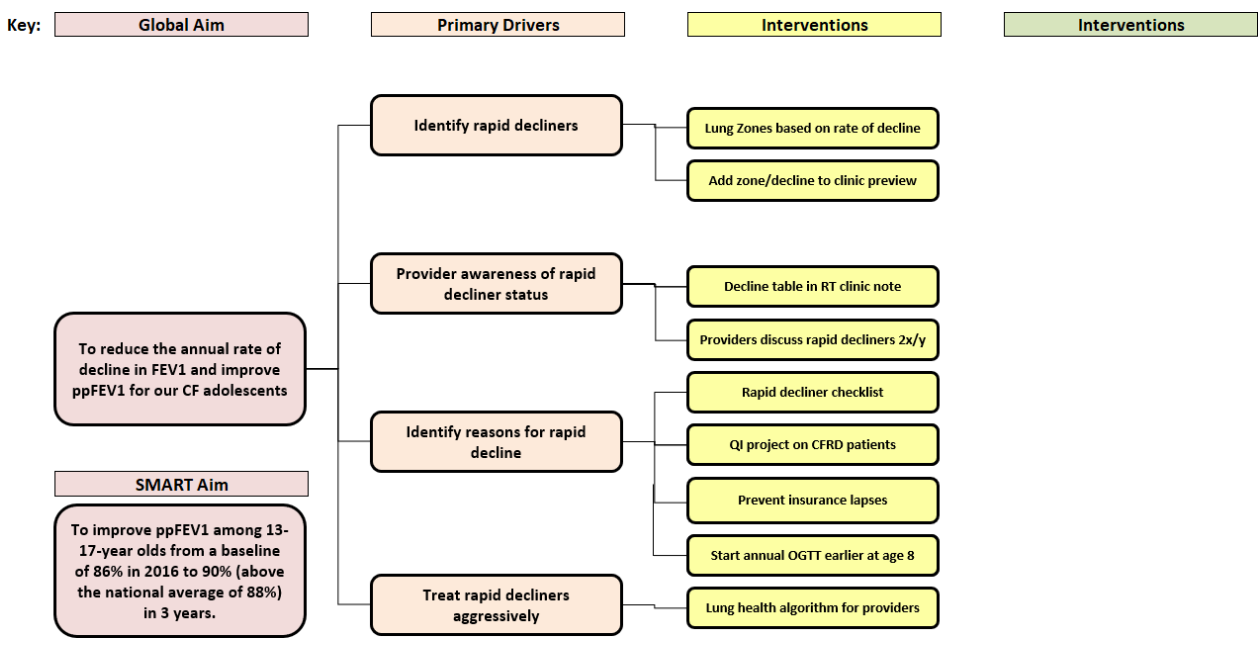

Figure 1 Driver diagram created to display factors that contribute to the achievement of our global aim. Further evaluation of rapid decliners revealed that CF-related diabetes and Medicaid insurance were risk factors for rapid decline in our adolescent population, which led to additional QI efforts. CF, cystic fibrosis; ppFEV 1 , per cent predicted forced expiratory volume in 1 s; QI, quality improvement; RT, respiratory therapist; CFRD, cystic fibrosis related diabetes; OGTT, oral glucose tolerance test. 


\begin{tabular}{|l|l|l|l|}
\hline Year & FEV Liters & $\begin{array}{l}\text { Baseline } \\
\text { ppFEV }\end{array}$ & $\begin{array}{l}\text { Rate of decline } \\
\text { in ppFEV }\end{array}$ \\
\hline 2013 & 1.6 & $106 \%$ & \\
\hline 2014 & 1.8 & $102 \%$ & $-4 \%$ \\
\hline 2015 & 1.9 & $94 \%$ & $-8 \%$ \\
\hline 2016 & 2.0 & $90 \%$ & $-4 \%$ \\
\hline $\begin{array}{l}\text { Lung Zone: D } \\
\text { Best FEV }\end{array}$ 2 2016: 2.1L, 91\% & \\
Average annual rate of decline: $-5 \% / y e a r$
\end{tabular}

Figure 2 Lung decline table included in the clinic note to highlight rate of decline and corresponding lung zone. FEV forced expiratory volume in $1 \mathrm{~s}, \mathrm{ppFEV}_{1}$, per cent predicted forced expiratory volume in $1 \mathrm{~s}$.

decline in $\operatorname{ppFEV}_{1}(-5.1 \%$ vs $-1.5 \% /$ year $)$, as did patients with Medicaid insurance compared with private insurance $(-3.5 \%$ vs $-1.2 \% /$ year $)$. These risk factors were therefore incorporated into our driver diagram and led to additional QI efforts at our programme. Lung zones were assigned based on approximate quartiles for our centre's 2013-2016 rate of decline in ppFEV $_{1}$. Many of our initiatives focused efforts on patients with accelerated decline in lung function (faster than average), who fall into the lowest two quartiles, the $\mathrm{C}$ and $\mathrm{D}$ zones, known as 'rapid decliners'.

A: no decline (or improvement).

B: decline of $0.01 \%-2.0 \% /$ year.

C: decline of $2.01 \%-3.5 \%$ /year.

D: decline $>3.5 \% /$ year.

The CF interdisciplinary team has a weekly clinic preview meeting to discuss patients with upcoming appointments in the next week. We added the 2016 ppFEV ${ }_{1}$ baseline, annual rate of decline and assigned lung zone to the weekly discussion sheet to highlight patients in zones C/D for provider awareness and team discussion at clinic preview.

\section{PDSA cycle 2/3 (February and March 2017)}

The CF RT created a lung decline table (figure 2), based off the lung function spreadsheet. This table was added to the RT clinic note for each patient, so the provider would not have to reference the lung function spreadsheet, making it easier to access and act on this information during clinic visits. We refined this cycle by encouraging providers to copy this table into their own clinic note to reference, also making it easier for an admitting hospital team to find the data and review.

\section{PDSA cycle 4 (April 2017)}

To encourage uniformity in care and focus attention on rapid decliners, we created a lung health algorithm for our providers (online supplemental figure 1), which includes an acute pulmonary exacerbation protocol. Based on the CF Foundation's benchmarking analysis of top-performing CF centres, it was found that more frequent follow-up and early, aggressive intervention of lung function decline were associated with improved outcomes. ${ }^{14}$ We used this to guide the creation of our algorithm to ensure rapid decliners received appropriate, step-up investigations and treatments and closer follow-up. The algorithm was available for providers to use on all patients but focused on those in the $\mathrm{C} / \mathrm{D}$ lung zones to align with the benchmarked best practices. The algorithm was discussed and agreed on by the full interdisciplinary team at a QI meeting.

\section{PDSA cycle 5/6 (April 2017)}

To identify potential reasons for individual lung function decline, we created a rapid decliner checklist (online supplemental figure 2). We determined that a checklist would be beneficial, as checklists play a vital role in improving patient safety and adherence to evidence-based best practices throughout the healthcare system. ${ }^{15}{ }^{16} \mathrm{~A}$ few members of the team met to create the checklist by brainstorming potential contributing factors to rapid decline with corresponding corrective actions and then reviewed it with the entire team to obtain feedback for improvement and achieve consensus.

The multidisciplinary team reviewed the checklist every 6 months during clinic preview for all patients in the C/D lung zones. We refined this cycle in 2018 to review the rapid decliner checklist annually during a QI meeting to ensure optimal provider attendance and involvement. Prior to the meeting, the providers receive the list of the patients in the $\mathrm{C} / \mathrm{D}$ lung zones and respond with comments about potential reasons for each patient's decline. The comments are reconciled into one document to discuss during the meeting and plan next step interventions for each patient.

\section{PDSA cycle 7 (November 2019)}

To improve our process of manually collecting annual baseline data to calculate the rate of decline, we partnered with our performance analytics team to create an automated report and scorecard. With this report, each patient's quarterly best $\mathrm{ppFEV}_{1}$ is directly pulled from our $\mathrm{CF}$ registry data and the annual baseline is automatically calculated. The scorecard includes quarterly and year-todate median $\mathrm{ppFEV}_{1}$ metrics for the entire CF programme with corresponding run charts. The scorecard is shared quarterly with the multidisciplinary CF team.

\section{MEASURES}

\section{Outcome measures}

Since this QI initiative is focused on improving lung function, we chose our clinical outcome measures to be the cohort's annual and quarterly median $\mathrm{ppFEV}_{1} . \mathrm{FEV}_{1}$ is defined as the maximum amount of air one can forcefully exhale in the first second. This value is converted to the per cent predicted, based off the individual's age, height, weight and race. ${ }^{17}$ We tracked these outcome measures for several groups: group aged 13-17 years, group aged 12-21 years and a longitudinal cohort of 49 adolescents. We tracked individual annual baseline $\mathrm{ppFEV}_{1}$ and individual average annual rate of decline in $\mathrm{ppFEV}_{1}$ as part of this project. We also tracked the per cent of 'rapid 
decliner' patients in the $\mathrm{C}$ and $\mathrm{D}$ zones each year as an additional outcome measure.

\section{Process measures}

Cycle-specific process measures are described in the PDSA results as follows.

\section{RESULTS}

PDSA cycle 1 (January 2017): calculated baseline lung function, created and assigned lung zones, added baseline, annual rate of decline and lung zone to the discussion sheet for clinic preview

To ensure our annual baselines could be accurately calculated from quarterly $\mathrm{FEV}_{1}$ data, we tracked the per cent of patients with a quarterly $\mathrm{FEV}_{1}$ missing. A quarterly $\mathrm{FEV}_{1}$ is defined as the best $\mathrm{FEV}_{1}$ that meets American Thoracic Society (ATS) standards during that quarter's clinic visit. We were then able to cross-reference any missing data with corresponding appointment data to validate. All (100\%) of those patients with a missing quarterly $\mathrm{FEV}_{1}$ truly did not have a PFT that quarter that met ATS standards. Of the adolescent patients for whom lung function data were available for the 2013-2016 time period $(n=69), 100 \%$ had a rate of decline calculated and a lung zone assigned. Twenty of the 69 patients transitioned to adult care during early 2017 due to the retirement of a provider or moved away; therefore, 49 patients continued to receive care at our programme throughout 2017 and were exposed to the interventions (our longitudinal cohort). The baseline, annual rate of decline and lung zone were added to the discussion sheet for clinic preview $100 \%$ of the time. The cycle was also studied via qualitative feedback at our weekly multidisciplinary CF team clinic preview and QI meetings to obtain input on the usefulness of the intervention from the team. The RT reported this process was easily integrated into her clinic preview workflow. However, we found that adding the baseline, annual rate of decline and lung zone to the clinic preview discussion sheet was not as helpful as expected, as some providers do not consistently attend the clinic preview meeting, and there was not always time during clinic preview to discuss the lung decline and zone. Additionally, the team often forgot to bring the discussion sheet to the clinic, making it hard to use the data and address the patient's rate of decline during the visit. The RT continued to include this information on the discussion sheet, but we refined this process by creating a table to include in the RT note, so providers had access to it during clinic.

\section{PDSA cycle 2/3 (February and March 2017): lung function decline table}

Copying the lung function decline table into the RT's note for the team to review allowed the providers to access it during outpatient clinics. The RT notes contained the lung function decline table for $98 \%$ of patients in 2017 for our longitudinal cohort of 49 patients. The RT qualitatively reported this was straightforward and efficient to add to her workflow. When the CF RT was out, we noted that the covering RTs did not consistently include the table. Thus, we worked toward developing a system where a single RT would serve as the back-up RT for CF clinic or the CF RT would prepare the tables before she was out. However, the location of the table in the RT note made it difficult for the outpatient providers and hospital providers to find the table during future encounters, as the RT notes are not as prominent as provider notes in our electronic medical record. Copying this table into the outpatient CF provider's note made it easier for clinic providers to find it and for an admitting team to review it on a hospital admission. Once the outpatient provider copies the table at the beginning of the year, it stays there for the remainder of the year, making it much easier to reference during clinic visits. The provider notes contained the lung function decline table $86 \%$ of the time in 2017. We did not require this, but most providers felt it was helpful and improved communication and completed it.

\section{PDSA cycle 4 (April 2017): lung health algorithm}

This algorithm encouraged consistency among providers by standardising education for patients and families, assessment for contribution factors of lung function decline, acute pulmonary exacerbation treatment, and chronic treatment and follow-up. Due to limited resources and our large patient population, we were not able to quantitatively track adherence to the algorithm. We studied this cycle by reviewing the lung health algorithm during QI meetings for qualitative feedback from the multidisciplinary team. We found that some aspects of the algorithm were difficult to implement, including rapid decliners not calling the clinic 5-7 days after starting antibiotics nor coming in for more frequent follow-up visits.

\section{PDSA cycle 5/6 (April 2017): rapid decliner checklist}

After the first year of this intervention, 54\% of rapid decliner $\mathrm{C} / \mathrm{D}$ zone patients had a checklist completed, and only one patient had two checklists completed. We found it difficult to review the checklist every 6 months during the interdisciplinary clinic preview meeting due to suboptimal provider attendance and the team forgetting to bring the checklists. Additionally, since the checklist review was dependent on patients having a scheduled appointment in the next week, then patients that cancelled their appointment would not have the opportunity to have their checklist completed until they rescheduled. Changing this process to review the checklist annually during the QI meeting resulted in greater provider attendance and ensured that all rapid decliners were discussed as a team (an improvement on PDSA 1). Allowing the providers to review the patients ahead of time allowed for efficient discussions.

\section{PDSA cycle 7 (November 2019): automated report and scorecard}

Creating a report that automatically pulls quarterly bests and baselines makes the process of calculating the annual 


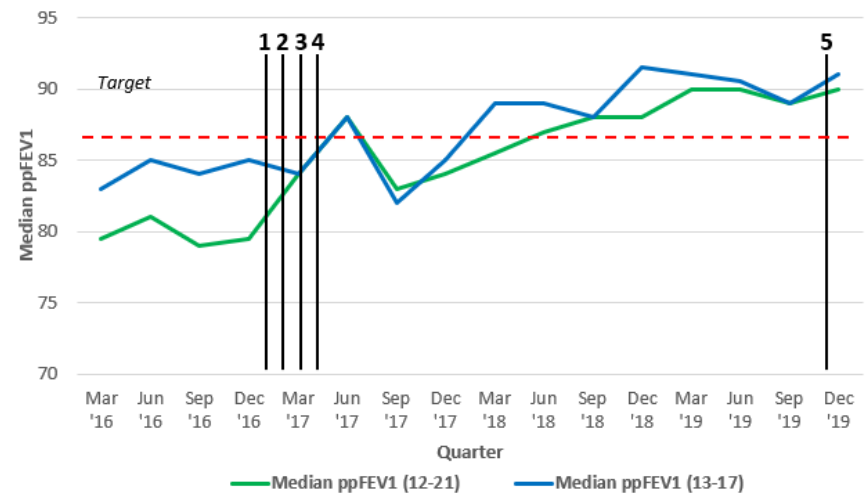

Figure 3 Median ppFEV, by quarter for adolescents. 1 (January 2017), SMART aim established; began putting baseline, rate of decline and lung zone into clinic preview document; began tracking quarterly median $\mathrm{ppFEV}_{1}$ for 13 17 and 12-21; 2 (February 2017), lung decline tables added to respiratory therapist note; 3 (March 2017), lung decline tables added to provider note; 4 (April 2017), rapid decline checklist implemented, lung health algorithm finalised; 5 (November 2019), automated report and scorecard finalised. ppFEV $_{1}$, per cent predicted forced expiratory volume in $1 \mathrm{~s}$.

rates of decline with corresponding lung zones faster due to the reduced manual component. The scorecard allows us to track our centre's quarterly and year-to-date median ppFEV $_{1}$ over time. The run charts promote easy data visualisation when sharing metrics with the $\mathrm{CF}$ team. The scorecard has run successfully for $83 \%$ of the quarters since initiation; we did not run the scorecard in quarter 2 of 2020 due to extremely limited in-clinic visits during the COVID-19 pandemic. Qualitative feedback on this report has been positive, and it has decreased the manual tracking burden on the $\mathrm{CF}$ team.

\section{Quantitative results}

To determine if our initiatives were successful, we tracked our outcome measure (median $\mathrm{ppFEV}_{1}$ ) quarterly by age group (13-17 and 12-21 years of age) in a crosssectional manner (figure 3 ). Our median $\mathrm{ppFEV}_{1}$ in both age groups showed substantial improvement over time, reaching our target of $90 \%$ in 2019 . We subsequently decided to expand these interventions outside of the original adolescent cohort to younger patients and to track the results by age group (including children 6-12 years of age).

We also looked at our longitudinal cohort of 49 patients 8-18 years of age as of 1 January 2013 who had at least one $\mathrm{ppFEV}_{1}$ value each year from 2013 to 2016 and received care at our institution in 2017 (exposed to the QI interventions). In this group, the median $\mathrm{ppFEV}_{1}$ increased from 81.3 to 83.3 in 2017 (figure 4). The mean rate of decline in $\mathrm{ppFEV}_{1}$ was $+0.7 \%$ in 2017 , and $45 \% \quad(\mathrm{n}=22)$ of the patients improved their baseline $\mathrm{ppFEV}_{1}$ with a median improvement of $+3.6 \%$. The mean annual rate of decline improved from $-2.1 \%$ /year for 2013-2016 to $-1.3 \%$ /year for $2014-2017,-1 \%$ /year for 2015-2018 and

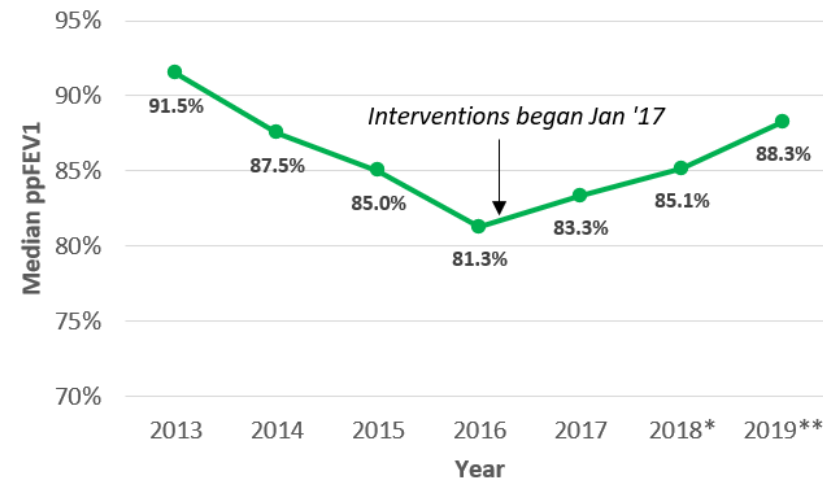

Figure 4 Annual median $\mathrm{ppFEV}_{1}$ for the longitudinal cohort of 49 adolescents with cystic fibrosis. ${ }^{*} n=46,{ }^{* *} n=40 . p p F E V_{1}$, per cent predicted forced expiratory volume in $1 \mathrm{~s}$.

-1\%/year for 2016-2019. Even though the size of the cohort continued to drop due to transitions into adult care, we continued to calculate median $\mathrm{ppFEV}_{1}$ and rates of decline for the longitudinal cohort in 2018 and 2019 and saw sustained improvements in median lung function for the remaining individuals. This longitudinal approach was unique because the same individuals were tracked over multiple years, and on average showed improvement in lung function instead of the gradual decline that would be expected with the natural course of CF lung disease progression. We also examined the per cent of patients in the $\mathrm{C}$ and $\mathrm{D}$ zones each year for this longitudinal cohort, and this measure of rapid decliners decreased overall from the start of the project (figure 5).

\section{DISCUSSION}

Among adolescent patients with CF, continually tracking individual annual baseline $\mathrm{ppFEV}_{1}$ and rate of lung function decline led to improved identification of rapid decliners and implementation of more aggressive treatments. In our patient cohorts, these interventions resulted in improvements in median lung function and reduction in mean rate of decline. If maintained, these changes are

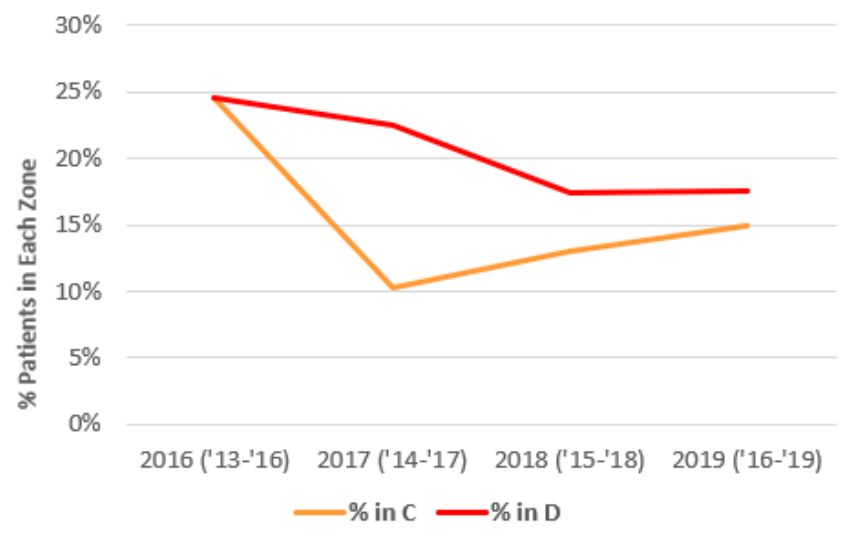

Figure 5 Per cent of adolescent patients with cystic fibrosis in the $\mathrm{C}$ and $\mathrm{D}$ lung decline zones (rapid decliners) each year ( $n=49$, longitudinal cohort). 
anticipated to result in slower progression of lung disease and increasing survival. These improvements may be attributable to a more standardised and proactive approach to decreases in lung function and increased clinician attention to patients with rapid decline, especially those with high baseline lung function whose decline could be more clinically silent. Of our interventions, we believe the lung function decline tables in the RT notes and annually discussing individualised reasons for rapid decline had the largest impact on improvement. This highlighted lung function decline for providers and allowed them to identify rapid decliners and intervene. A lesson learnt was that these interventions require provider and team buy-in and involvement to be successful. We were lucky to have excellent team buy-in from the beginning of our project. Our team was very motivated to improve after learning that our programme's lung function was below average in 2014 and 2015; we feel this buy-in contributed to our successes. We found benchmarking top-performing centres extremely beneficial, as it stimulated these QI initiatives and allowed us to choose appropriate goals. We also learnt the importance of coproduction with our patients and families. Although we did not have patient involvement at the time we designed this project, we now have two parent partners who are active members of our QI team. As a next step, we are expanding these interventions beyond the original cohort of teens to include all patients with at least 2 years of PFT data. Although few patients in our studied cohort were on highly effective CFTR modulator medications, the sustainability of these interventions will have to be modified due to the expansion in modulator therapy availability for $\mathrm{CF}$ and the corresponding increase in baseline lung function. Once patients reach their new baseline lung function on highly effective modulator therapy, we will be able to meaningfully track rate of decline again.

Acknowledgements The authors thank the entire Emory+Children's Cystic Fibrosis multidisciplinary care team for their support of this project.

Contributors MS and RWL collected the data. SS and RWL analysed and interpreted the data. SS and RWL drafted the article. MS provided critical revision of the article. All authors conceptualised and designed the work and approved the final version to be published.

Funding This quality improvement initiative was funded with salary support for MS and RWL from the Emory Cystic Fibrosis Foundation Care Center Grant (CFF CCO02).

Competing interests RWL and MS report grant salary support from the Cystic Fibrosis Foundation during the conduct of this study. RWL reports grants from the Cystic Fibrosis Foundation and Vertex Pharmaceuticals Incorporated and serving on advisory boards for Vertex Pharmaceuticals Incorporated, outside of the submitted work. SS reports no conflicts of interest.

Patient consent for publication Not required.

Ethics approval The Children's Healthcare of Atlanta's institutional review board determined the project was not a research activity and the initiative was given a not-human research determination.

Provenance and peer review Not commissioned; externally peer reviewed.
Data availability statement Due to the nature of this research, participants of this study did not agree for their data to be shared publicly, so supporting data is not available. No data are available.

Supplemental material This content has been supplied by the author(s). It has not been vetted by BMJ Publishing Group Limited (BMJ) and may not have been peer-reviewed. Any opinions or recommendations discussed are solely those of the author(s) and are not endorsed by BMJ. BMJ disclaims all liability and responsibility arising from any reliance placed on the content. Where the content includes any translated material, BMJ does not warrant the accuracy and reliability of the translations (including but not limited to local regulations, clinical guidelines, terminology, drug names and drug dosages), and is not responsible for any error and/or omissions arising from translation and adaptation or otherwise.

Open access This is an open access article distributed in accordance with the Creative Commons Attribution Non Commercial (CC BY-NC 4.0) license, which permits others to distribute, remix, adapt, build upon this work non-commercially, and license their derivative works on different terms, provided the original work is properly cited, appropriate credit is given, any changes made indicated, and the use is non-commercial. See: http://creativecommons.org/licenses/by-nc/4.0/.

\section{REFERENCES}

1 Elborn JS. Cystic fibrosis. The Lancet 2016;388:2519-31.

2 Sanders DB, Bittner RCL, Rosenfeld M, et al. Pulmonary exacerbations are associated with subsequent FEV ${ }_{1}$ decline in both adults and children with cystic fibrosis. Pediatr Pulmonol 2011;46:393-400.

3 Liou TG, Adler FR, FitzSimmons SC, et al. Predictive 5year survivorship model of cystic fibrosis. Am J Epidemiol 2001;153:345-52.

4 Britto MT, Kotagal UR, Hornung RW, et al. Impact of recent pulmonary exacerbations on quality of life in patients with cystic fibrosis. Chest 2002;121:64-72.

5 Waters V, Stanojevic S, Atenafu EG, et al. Effect of pulmonary exacerbations on long-term lung function decline in cystic fibrosis. European Respiratory Journal 2012;40:61-6.

6 Johnson C, Butler SM, Konstan MW, et al. Factors influencing outcomes in cystic fibrosis: a center-based analysis. Chest 2003;123:20-7.

7 Schechter MS, Schmidt HJ, Williams R, et al. Impact of a program ensuring consistent response to acute drops in lung function in children with cystic fibrosis. J Cyst Fibros 2018;17:769-78.

8 Cystic Fibrosis Foundation. 2018 patient registry annual data report, 2020. Available: https://www.cff.org/Research/ResearcherResources/Patient-Registry/2018-Patient-Registry-Annual-DataReport.pdf

9 VandenBranden SL, McMullen A, Schechter MS, et al. Investigators and coordinators of the epidemiologic study of cystic fibrosis. lung function decline from adolescence to young adulthood in cystic fibrosis. Pediatr Pulmonol 2012;47:135-43.

10 Morgan WJ, Wagener JS, Yegin A, et al. Probability of treatment following acute decline in lung function in children with cystic fibrosis is related to baseline pulmonary function. $J$ Pediatr 2013;163:1152-7.

11 Moran A, Brunzell C, Cohen RC, et al. Clinical care guidelines for cystic fibrosis-related diabetes: a position statement of the American diabetes association and a clinical practice guideline of the cystic fibrosis Foundation, endorsed by the pediatric endocrine Society. Diabetes Care 2010;33:2697-708.

12 Institute for Healthcare Improvement. How to improve: IHI. Available: http://www.ihi.org/resources/Pages/Howtolmprove/default.aspx

13 Harun SN, Wainwright C, Klein K, et al. A systematic review of studies examining the rate of lung function decline in patients with cystic fibrosis. Paediatr Respir Rev 2016;20:55-66.

14 Boyle MP, Sabadosa KA, Quinton HB, et al. Key findings of the US Cystic Fibrosis Foundation's clinical practice benchmarking project. BMJ Qual Saf 2014;23:i15-22.

15 Birk S. Lists that work: the healthcare leader's role in implementation. Healthc Exec 2013;28:28-30.

16 Winters BD, Gurses AP, Lehmann H, et al. Clinical review: checklists - translating evidence into practice. Crit Care 2009;13:210-9.

17 Graham BL, Steenbruggen I, Miller MR, et al. Standardization of spirometry 2019 update. An official American thoracic Society and European respiratory Society technical statement. Am J Respir Crit Care Med 2019;200:e70-88. 